\title{
Neutrophil-to-lymphocyte Ratio and Platelet-to- lymphocyte Ratio in the Prediction of Pancreatic Cancer Patients' prognosis: a retrospective study
}

\section{Yungu Chen}

Shanghai Jiao Tong University School of Medicine Affiliated Renji Hospital

\section{Lek Man Lam}

Shanghai Jiao Tong University School of Medicine

Lina $\mathrm{He}$

Shanghai Jiao Tong University School of Medicine

Yiu Sing Tsang

Shanghai Jiao Tong University School of Medicine

Qing Xia ( $\square$ jingtianxq@163.com )

Shanghai Jiao Tong University School of Medicine Affiliated Renji Hospital

\section{Research article}

Keywords: neutrophil-to-lymphocyte ratio, platelet-to-lymphocyte ratio, pancreatic cancer, prognosis, gemcitabine, 5-fluorouracil

Posted Date: June 24th, 2019

DOI: https://doi.org/10.21203/rs.2.10553/v1

License: (c) (1) This work is licensed under a Creative Commons Attribution 4.0 International License. Read Full License 


\section{Abstract}

Background Although neutrophil-to-lymphocyte ratio (NLR) and a high platelet-to-lymphocyte ratio (PLR) have been reported to be an inverse prognostic predictor of survival in patients with pancreatic cancer $(\mathrm{PC})$, the comparison of their prognostic roles in patients with PC undergoing gemcitabine-based chemotherapy and 5-fluorouracil (5-FU) remains unclear. Methods This study was designed and performed to determine the predictive role of NLR and PLR in patients diagnosed with PC who underwent one of these two regimens. We retrospectively enrolled 95 patients diagnosed with PC undergoing supportive care, gemcitabine-based chemotherapy or 5-FU therapy from January 2015 to October 2018. Univariate and multivariate Cox regression analyses were done to identify clinicopathological predictors of time to treatment failure (TTF) and overall survival (OS), including pretreatment NLR and PLR. Results Pretreatment NLR was significantly associated with metastasis. Pretreatment NLR was an independent prognostic predictor of both TTF and OS for patients with PC, with NLR>4.0 predicting worse survival. PLR, however, didn't independently predict TTF or OS. There were no significant difference in the OS of patients undergoing gemcitabine-based regimens and 5-FU regimens when divided into two subgroups: NLR $\leq 4.0$ and $>4.0$. Conclusions Pretreatment NLR is a promising independent outcome predictor for patients with PC. The predictive value of PLR might not be as good as NLR. NLR might not be a suitable factor in the selection of regimens for patients with PC in term of gemcitabine and 5-FU.

\section{Introduction}

Pancreatic cancer (PC) is one of the most lethal cancers that remains a challenging medical problem for many years. The most common form is pancreatic ductal adenocarcinoma (PDA), the tenth most common solid cancer and the fourth leading cause of death from cancer in the United States.[1] The prognosis of PC is very poor, with the 5-year survival rate of only 7\%.[2] Unfortunately, there are no specific symptoms. Patients at early stage are usually symptom-free, and PC in those who present with unspecific abdominal discomfort, weight loss or more specific jaundice are already in advanced stage. Most patients are diagnosed with metastatic disease and few show a sustained response to chemo- or radiation therapy.[3]

Considering the poor prognostic outcome of patients with PC, efforts have been made to explore the predictive factors of this malignancy. It has been recognized that inflammatory responses play decisive roles at different stages of tumor development [4], and several inflammatory biomarkers have been proposed for the evaluation of cancer patients. Recently, pretreatment neutrophil-to-lymphocyte ratio (NLR) has been revealed to be a promising prognostic predictor for pancreatic cancer by several studies, where low NLR stands for better survival in patients with pancreatic cancer.[ 5,6$]$ NLR is calculated by neutrophil count divided by lymphocyte count, which can be easily obtained by routine blood tests. Previous studies indicated that a high level of NLR might be significantly associated with poorer prognosis of several tumors, including colorectal cancer, lung cancer, gastric cancer, esophageal cancer and so on[ 7-9]. There are also evidences suggesting that platelet-to-lymphocyte (PLR), as another easily accessible biomarker that is calculated by platelet count divided by lymphocyte count, may also be a 
prognostic predictor of a variety of malignancies such as ovarian cancer[ 10], breast cancer[ 11] and nonsmall-cell lung cancer[12], where low PLR suggests a better outcome.

This study was to determine the association between both pretreatment NLR and PLR and survival of patients with PC treated with supportive care, gemcitabine-based chemotherapy or 5-FU therapy.

\section{Methods}

\section{Patients and treatment}

Searching the database system of electronic medical charts, we collected clinical data of patients with the diagnosis of pancreatic cancer referred to Renji Hospital affiliated to Shanghai Jiao Tong University School of Medicine between January 2015 and October 2018. Patients whose diagnosis was PC were included in the study. Patients without available data or those with infection, regimens with steroids or aspirin, autoimmune disease or other conditions that might possibly confounded neutrophil count, lymphocyte count or platelet were excluded. In this study, patients underwent supportive care, gemcitabine-based therapy or 5-FU therapy. Gemcitabine-based therapy included gemcitabine monotherapy and gemcitabine combination therapy. Treatment was not terminated until tumor recurrence, tumor progression, treatment toxicity or patients' requirement for withdrawal. The doses and regimens were adjusted by corresponding physicians in accordance with adverse events and general conditions of the individual patient.

\section{Clinical and laboratory data collection}

Patients included were either chemotherapy-naïve or chemotherapy-free for at least 1 month before they were referred to Renji Hospital affiliated to Shanghai Jiao Tong University. Baseline data was collected before treatment start. The history of patients was taken on the first days of hospitalization. Karnofsky performance status (KPS) was evaluated by treating physicians before the commencement of chemotherapy. Tumor location and TNM (tumor, lymph node and metastasis) staging were judged from the result of computed tomography (CT) or positron emission tomography (PET) in accordance with the $7^{\text {th }}$ edition of the AJCC Cancer Staging Manual. Biological markers, including carbohydrate antigen 19-9 (CA 19-9), carcinoembryonic antigen (CEA), carbohydrate antigen-125 (CA-125), neutrophil count, lymphocyte count and platelet count were collected within 3 weeks before the first cycle of chemotherapy as the pretreatment data. NLR was defined by the absolute neutrophil count divided by the absolute lymphocyte count. Likewise, PLR was defined by the absolute platelet count divided by the absolute lymphocyte count. All consecutive parameters were categorized for the further analysis as follows: age ( $\leq 65$ or $>65$ years), body mass index (BMI) $(\leq 18.5,18.5-24.0$ or $>24.0)$, CA $19-9(\leq 1000$ or $>1000 \mathrm{U} / \mathrm{ml})$, CEA ( $\leq 5$ or $>5 \mathrm{ng} / \mathrm{ml})$, CA-125 ( $\leq 38$ or $>38 \mathrm{U} / \mathrm{ml})$. Cut-off values were set on the basis of previous studies.[13,14] 


\section{Statistical analysis}

The $\chi^{2}$ test or Fisher's exact test were used to compare baseline patient characteristics that were categorized variables. The association between pretreatment NLR and the time to treatment failure (TTF) as well as overall survival (OS) was evaluated, so was pretreatment PLR. TTF was defined as the time from the date of chemotherapy initiation to the date of termination due to various reasons, including tumor recurrence, tumor progression, treatment toxicity or patients' requirement for withdrawal. If a patient had not reached the endpoint caused by any of these reasons, TTF was censored at the time of the last follow-up. Recurrence and progression were determined using CT or PET. OS was calculated from the date of chemotherapy initiation to the date of death for any reason, or censored at the date of the last follow-up if the endpoint event was not observed. Univariate and multivariate Cox regression analyses were done to identify clinicopathological predictors of TTF and OS, including age, gender, BMI, KPS, personal history, diabetes at diagnosis, tumor location, TNM stage, CA 19-9, CEA, CA-125 and pretreatment NLR and PLR. The differences of TTF and OS were compared utilizing the Kaplan-Meier method with log-rank tests for survival plot depiction and Cox-regression analysis for the evaluation of hazard ratio (HR) and its 95\% confidence interval $(95 \% \mathrm{Cl})$.

In order to set the cut-off points of both NLR and PLR, Receiver Operating Curves (ROC) were depicted. The classification variable was long vs short-term survival (>6 vs $\leq 6$ months). For one thing, the choice of the 6 months as the division point was due to the convenience of the study, since a relevant proportion

of patients (47.4\%) were classified as short-term survivors and only 4 patients had a follow-up less than 6 months. For another, the majority of patients included in this study was in stage IV, whose median survival was only 4-6 months[ 15], so the choice of the 6 months was feasible. The effects of potential prognostic predictors were tested by Cox regression. Only variables with a statistical significance in univariate analysis were investigated in multivariate analysis.

The statistical significance of all tests was two-sided, $p<0.05$. Analyses and calculation were done by IBM SPSS Statistics 24.0.

\section{Regulatory consideration}

This study is approved by the Ethic Committee of Renji Hospital affiliated to Shanghai Jiao Tong University.

\section{Results}

A total of 103 patients diagnosed with pancreatic cancer were referred to Renji Hospital affiliated to Shanghai Jiao Tong University School of Medicine between January 2015 and October 2018. All patients were histologically confirmed to have PC by biopsy, from which were excluded 4 patients with unavailable 
data and 4 patients who had infections, steroid regimens or autoimmune disease at the time of baseline data collection. 95 patients were ultimately eligible for the inclusion.

The demographics and characteristics of all patients were shown in table 1. Among the 95 patients in the study, 56 were male and 39 were female. The median of age at baseline was 62 years (range, 42-83). BMI ranged from 14.4 to $28.1 \mathrm{~kg} / \mathrm{m}^{2}$, with the median of $20.8 \mathrm{~kg} / \mathrm{m}^{2} .42 .1 \%$ patients had KPS $\leq 80$. As for the investigation of risk factors of PC, $32.6 \%$ of patients had a history of smoking while $16.8 \%$ had a history of alcohol intake. $13.7 \%$ of patients had a family history of cancer. $32.6 \%$ patients had diabetes at the time of PC diagnosis. Histologically, $87.4 \%$ patients were confirmed to have PC while others had neuroendocrine tumor and mucinous carcinoma. Tumor in $42.1 \%$ patients located in the head of pancreas and distantly metastasized in $72.6 \%$ patients. Routine biochemical tests performed before the commencement of treatment were collected as baseline data, some of which were long recognized prognostic predictors. In term of therapy, $12.6 \%$ patients underwent supportive care, whereas $53.7 \%$ patients were treated by gemcitabine-based therapy and $33.7 \%$ patients by 5 -FU. The median neutrophil count was $3.60 * 10^{9} / \mathrm{L}$, with the range of $1.44-15.75 * 10^{9} / \mathrm{L}$. The median lymphocyte count was $1.42 * 10^{9} / \mathrm{L}$, ranging from $0.50 * 10^{9}$ to $4.50 * 10^{9} / \mathrm{L}$. Median NLR was 2.6 (range, $0.5-18.0$ ) with $27(28.4 \%)$ patients having NLR>4.0 and that of PLR was 148 (range, 51-785) with $39(41.1 \%)$ patients having PLR>169. Among all 95 patients, 39 (41.1\%) presented with the biomarker CA 19-9>1000 U/ml, 47 (49.5\%) with CEA $>5 \mathrm{ng} / \mathrm{ml}$ and 49 (51.6\%) with CA-125>38 U/ml.

As of October 2018, 72 patients reached TTF endpoint and 45 patients reached OS endpoint. For better utility of NLR as a predictor for the prognosis of patients with PC, a ROC curve was constructed to find the cut-off point of NLR. The areas under curve (AUC) was 0.754. A fixed cut-off value of 4.0 was taken for the analysis, yielding a sensitivity of $80.0 \%$ and a specificity of $60.0 \%$ (figure 1 ). With NLR=4.0 as the cutoff point, it was confirmed that pretreatment NLR was significantly associated with TTF, with median TTF of 8.0 months and 2.0 months for patients with NLR $\leq 4.0$ and $>4.0$ respectively, $\mathrm{HR}=3.158(95 \% \mathrm{Cl}, 1.805$ 5.527), $p=0.0001$ (figure 2). NLR was also significantly associated with OS. The median OS of patients with NLR $\leq 4.0$ was 21.0 months and that of patients with NLR>4.0 was 5.0 months, $H R=4.090$ (HR=2.073-8.071), $p=0.0001$ (figure 2). A ROC curve was also constructed to find the appropriate cut-off point. The AUC was 0.645 . PLR=169 was found to be the best cut-off point in this study, with a sensitivity of $53.3 \%$ and a specificity of $70.0 \%$ (figure 1). Median TTF of PLR $\leq 169$ and $>169$ was 6.0 months and 3.0 months respectively, $\mathrm{HR}=1.511$ ( $95 \% \mathrm{Cl}, 0.940-2.431$ ), $p=0.088$ (figure 3 ). And median OS of PLR $\leq 169$ and $>169$ was 17.0 months and 10.0 months respectively, $\mathrm{HR}=1.683(95 \% \mathrm{Cl}, 0.917-3.089), p=0.089$ (figure 3). The associations between NLR subgroups and biomarker CA 19-9 ( $p=0.672)$, CEA $(p=0.455)$ and CA-125 ( $p=0.625$ ) were found not of significance (table 1$)$.

It was uncovered that NLR was significantly associated with $M$ stage, where NLR $>4.0$ suggested a more likely metastatic scenario $(p=0.001)$. And in those patients with localized tumors at the time of diagnosis, their NLR was almost all below $4.0(25 / 26)$. Such an association might partially explain the association between NLR and prognosis, for patients with metastatic PC undoubtably tend to have a worse 
prognosis. Nevertheless, PLR was not found to be significantly associated with tumor metastasis $(p=0.211)$.

To evaluate whether NLR and PLR were independent predictors of prognosis, univariate and multivariate Cox-regression analyses for both TTF and OS were performed (table 2). In the assessment of potential prognostic variables of TTF, N stage, M stage, CA 19-9, CA-125, CEA, pretreatment NLR were found to be significantly associated with TTF at univariate Cox-regression analysis. At multivariate analysis, only NLR had statistically significant association with TTF, the HR of NLR being 3.158 (95\% Cl, 1.805-5.527), $p=0.0001$, although $\mathrm{M}$ stage exhibited a marginal significance $(p=0.051)$. At univariate analysis of $0 \mathrm{~S}$, it was found that $N$ stage, $M$ stage, CA 19-9, CA-125, CEA, pretreatment NLR were significantly associated with OS. NLR was proved to be an independent prognostic factor of OS, HR being $4.090(95 \% \mathrm{Cl} 2.073-$ 8.071). $p=0.0001$. Meanwhile $\mathrm{N}$ stage also presented a statistical significance $(p=0.012)$. In univariable analysis, pretreatment PLR didn't show any significant association with TTF (HR=1.511 [0.940-2.431], $p=0.088$, figure 3 ) or OS (HR=1.683[0.917-3.089], $p=0.089$, figure 3 ).

Among the 83 patients selected for the study who underwent chemotherapy, the regimens of 51 patients was gemcitabine-based regimens and that of the others was 5-FU containing regimens. Gemcitabinebased therapy displayed a minor superior OS when compared to 5-FU, with median OS of 17.0 months and 15.0 months respectively (HR=1.525[0.776-2.995]). However, this was not of statistical significance ( $p=0.217$, figure 4). To evaluate the influence of NLR on the type of chemotherapy, the association between chemotherapy and OS was analyzed by separating patients into low and high pretreatment NLR. No significant interaction was found, with no difference between gemcitabine-based and 5-FU in the low NLR subgroup ( $p=0.310)$ and the high NLR subgroup ( $p=0.409)$.

\section{Discussion}

NLR has been confirmed to be a prognostic predictor in various cancers that significantly correlates with response rates, therapeutic effects and survival rates.[ 7-9] For patients with pancreatic cancer, high pretreatment NLR is found to be an unfavorable predictor of OS and TTF, and some studies pointed out that patients with high pretreatment NLR would tend to have a longer OS or PFS if their NLR was lowered after the initiation of chemotherapy.[16,17] In the present study, we demonstrated that pretreatment NLR independently predicted the prognosis of patients with advanced or localized PC, even after adjusting for potential confounding factors. But PLR failed to independently predict the prognostic outcome in term of both TTF and OS.

A number of studies have suggested the inverse association between both NLR and PLR and prognosis of patients with PC. $[6,18]$ Although it has long been established that NLR and PLR both play a role in predicting the prognosis of various malignancies, the question which one is more predictive and suggestive is still debatable. The comparison between NLR and PLR was done to analyze which factor does better in predicting the prognosis of patients with PC. 
Inflammation is a hallmark of various cancers.[19] Neutrophilia is a common phenomenon in PC. Multiple mechanisms have been hypothesized or demonstrated. It was proposed that neutrophil might aid metastasis of pancreatic cancer because it is able to mediate epithelial-mesenchymal transition of cancer cells by secreting elastase.[20] Neutrophil can secrete vascular endothelial growth factor (VEGF) and matrix metalloproteinase 9 , which promote angiogenesis thus facilitating the growth and metastases of PC.[21] Besides, neutrophil may help with the creation of immunosuppressive microenvironment of pancreatic cancer by suppressing CD8+T cells.[22] Pancreatic cancer is also associated with declined lymphocyte count. The proliferation of Lymphocytes is suppressed by various immunosuppressive cytokines during tumorigenesis, principally including transforming growth factor $\beta$ (TGF- $\beta$ ) and interleukin 10 (IL-10).[23] The association between thrombocytosis and PC is still not well understood. Platelets can secrete tumor growth factors, such as VEGF, TGF- $\beta$, platelet-derived growth factor, and insulin-like growth factor-1, which play a critical role in cancer angiogenesis and metastasis.[24]

In this study, NLR did provide independent prognostic information of survival in term of both TTF and OS, whereas PLR was not significantly associated with TTF or OS. Several studies have compared the predictive value of NLR and PLR in term of survival, response rates to treatment and recurrence after resection. NLR was recognized as a promising prognostic predictor whereas the association of PLR with patient outcome seems to be controversial.[25] Hasegawa et al. reported that pretreatment NLR might be a useful predictive marker for the pathological response to preoperative therapy in pancreatic cancer patients, which pretreatment PLR failed to predict.[26] Martin et al. found both NLR and PLR were predictive of overall survival of patients with advanced $P C$, despite a more powerful predictive value of NLR (HR=1.81, $p=0.0007)$ than PLR (HR=1.64, $p=0.007)$. [27] Stotz et al. reported that NLR added independent prognostic information to other well established prognostic factors in patients with pancreatic cancer, regardless of the undergoing therapeutic modality $(H R=2.532, p<0.001)$, but PLR did not show any predictive value as for overall survival in patients with both inoperative $(p=0.612)$ and operative pancreatic cancer $(p=0.458)$. [28] Yang et al performed a meta-analysis and found that high peripheral blood PLR suggested a poor prognosis for patients with pancreatic cancer.[29] Kishi et al analyzed 65 patients with pancreatic cancer and drew the conclusion that PLR was not associated with the prognosis of these patients.[ 30] As far as this study is concerned, PLR failed to show the same significant association as NLR did. NLR might possess a better predictive value than PLR does in patients with PC.

5-FU regimens were in common use prior to the adoption of gemcitabine as the chemotherapy for patients with PC. Nonetheless, according to several phase II and randomized controlled trials, both bolus and infusion leucovorin modulated 5-FU-containing regimens are associated with low response rates and survival inferior to that seen with gemcitabine.[31, 32] Still, 5-FU remains an alternative for gemcitabineintolerant patients. In this study, when patients were subgrouped by the criterion of NLR $\leq 4.0$ and $>4.0$, the OS did not significantly vary between gemcitabine-based regimens and 5-FU regimens. The comparison of NLR's prognostic roles in patients with PC undergoing gemcitabine-based chemotherapy and 5-FU remains unclear, and there was no significant difference in the OS of patients undergoing gemcitabinebased regimens and 5-FU regimens when divided into two subgroups: NLR $\leq 4.0$ and $>4.0$. NLR might not 
be a suitable factor in the selection of regimens between gemcitabine-based regimens and 5-FU for patients with PC.

We recognize several limitations in our study. First, the research was retrospective with a relatively small sample size. Besides, the analysis of NLR and PLR for predictive values of different chemotherapeutic modalities was achieved by dividing further the population into smaller chemotherapy subgroups. Moreover, the major drawback is that treatment assignment was not randomized. Finally, blood samples were not derived during follow-up, making it infeasible to analyze whether further change of NLR and PLR would predict prognosis more accurately.

\section{Conclusions}

In conclusion, pretreatment NLR is a promising independent outcome predictor for patients with pancreatic cancer. The predictive value of PLR might not be as good as NLR. NLR might not be a suitable factor in the selection of regimens for patients with PC in term of gemcitabine and 5-FU.

\section{List Of Abbreviations}

NLR: neutrophil-to-lymphocyte ratio

PLR: platelet-to-lymphocyte ratio

PC: pancreatic cancer

PDA: pancreatic ductal adenocarcinoma

5-FU: 5-fluorouracil

TTF: time to treatment failure

OS: overall survival

HR: hazard ratio

Cl: confidence interval

ROC: receiver operating curves

KPS: Karnofsky performance status

CT: computed tomography

PET: positron emission tomography 
CA 19-9: carbohydrate 19-9

CEA: carcinoembryonic antigen

CA-125: carbohydrate antigen-125

BMI: body mass index

VEGF: vascular endothelial growth factor

TGF- $\beta$ : transforming growth factor $\beta$

\section{Declarations}

\section{Ethics approval and consent to participate}

This study is approved by the Ethic Committee of Renji Hospital affiliated to Shanghai Jiao Tong University. Patients' consent was obtained.

\section{Consent for publication}

Not applicable

\section{Availability of data and material}

The datasets used and/or analysed during the current study are available from the corresponding author on reasonable request.

\section{Competing interests}

The authors declare that they have no competing interests.

\section{Funding}

This study was supported by Young Scientist Project of the National Natural Science Foundation of China (no. 81702843). The funder had no role in the study except for financial support.

\section{Authors' contributions}


YGC, LKL, LNL, YST and QX designed the idea and wrote the manuscript. YGC, LKL, LNH and YST collected the data and participated in the design of the study. All authors read and approved the manuscript.

\section{Acknowledgements}

Not applicable

\section{References}

1. Siegel RL, Miller KD, Jemal A: Cancer Statistics, 2017. CA Cancer J Clin 2017, 67(1):7-30.

2. Miller KD, Siegel RL, Lin CC, Mariotto AB, Kramer JL, Rowland JH, Stein KD, Alteri R, Jemal A: Cancer treatment and survivorship statistics, 2016. CA Cancer J Clin 2016, 66(4):271-289.

3. Yachida S, Jones S, Bozic I, Antal T, Leary R, Fu B, Kamiyama M, Hruban RH, Eshleman JR, Nowak MA et al: Distant Metastasis Occurs Late during the Genetic Evolution of Pancreatic Cancer. Nature 2010, 467(7319):1114-7.

4. Grivennikov SI, Greten FR, Karin M: Immunity, inflammation, and cancer. Cell 2010, 140(6):883-899.

5. Cheng H, Long F, Jaiswar M, Yang L, Wang C, Zhou Z: Prognostic role of the neutrophil-to-lymphocyte ratio in pancreatic cancer: a meta-analysis. Scientific Reports 2015, 5:11026.

6. Zhou Y, Wei Q, Fan J, Cheng S, Ding W, Hua Z: Prognostic role of the neutrophil-to-lymphocyte ratio in pancreatic cancer: A meta-analysis containing 8252 patients. Clinica chimica acta; international journal of clinical chemistry 2018, 479:181.

7. Li M-X, Liu X-M, Zhang X-F, Zhang J-F, Wang W-L, Zhu Y, Dong J, Cheng J-W, Liu Z-W, Ma L et al: Prognostic role of neutrophil-to-lymphocyte ratio in colorectal cancer: A systematic review and metaanalysis. International Journal of Cancer 2014, 134(10):2403-2413.

8. Cedrés S, Martínez A, Martinez P, Navarro A, Zamora E, Mulet-Margalef N, Felip E: Neutrophil to lymphocyte ratio (NLR) as an indicator of poor prognosis in stage IV non-small cell lung cancer. Clinical and Translational Oncology 2012, 14(11):864-869.

9. Yang X, Huang Y, Feng JF, Liu JS: Prognostic significance of neutrophil-to- lymphocyte ratio in esophageal cancer: a meta-analysis. Oncotargets \& Therapy 2015, 8(default):789.

10. Supachai R, Siriwan T, Sumonmal M, Sunamchok S, Thaovalai T: Platelet to lymphocyte ratio as a prognostic factor for epithelial ovarian cancer. Journal of Gynecologic Oncology 2012, 23(4):265-273. 
11. Krenn-Pilko S, Langsenlehner U, Thurner EM, Stojakovic T, Pichler M, Gerger A, Kapp KS, Langsenlehner T: The elevated preoperative platelet-to-lymphocyte ratio predicts poor prognosis in breast cancer patients. British Journal of Cancer 2014, 110(10):2524.

12. Nikolić I, Kukulj S, Samaržija M, Jeleč V, Žarak M, Orehovec B, Taradi I, Romić D, Kolak T, Patrlj L: Neutrophil-to-lymphocyte and platelet-to-lymphocyte ratio help identify patients with lung cancer, but do not differentiate between lung cancer subtypes. Croatian Medical Journal 2016, 57(3):287-292.

13. Xue P, Kanai M, Mori Y, Nishimura T, Uza N, Kodama Y, Kawaguchi Y, Takaori K, Matsumoto S, Uemoto S: Neutrophil-to-lymphocyte ratio for predicting palliative chemotherapy outcomes in advanced pancreatic cancer patients. Cancer Medicine 2014, 3(2):406-415.

14. Krishnan S, Vishal Rana, Janjan NA, Abbruzzese JL, Gould MS, Das P, Delclos ME, Shana Palla MS, PhD SGM, Gauri Varadhachary MD: Prognostic factors in patients with unresectable locally advanced pancreatic adenocarcinoma treated with chemoradiation. Cancer 2010, 107(11):2589-2596.

15. Vincent A, Herman J, Schulick R, Hruban RH, Goggins M: Pancreatic cancer. The Lancet 2011, 378(9791):607-620.

16. Formica V, Morelli C, Ferroni P, Nardecchia A, Tesauro M, Pellicori S, Cereda V, Russo A, Riondino S, Guadagni F: Neutrophil/lymphocyte ratio helps select metastatic pancreatic cancer patients benefitting from oxaliplatin. Cancer Biomarkers 2016, 17(3):335.

17. Luo G, Guo M, Liu Z, Xiao Z, Jin K, Long J, Liu L, Liu C, Xu J, Ni Q: Blood Neutrophil-Lymphocyte Ratio Predicts Survival in Patients with Advanced Pancreatic Cancer Treated with Chemotherapy. Annals of Surgical Oncology 2015, 22(2):670-676.

18. Xu ZS, Zhang FP, Zhang Y, Ou-Yang YP, Yu XW, Wang WL, Xu WJ, Luo ZQ: Prognostic role of the pretreatment platelet-lymphocyte ratio in pancreatic cancer: a meta-analysis. Oncotarget 2017, 8(58):9900399012.

19. O'Byrne KJ, Dalgleish AG: Chronic immune activation and inflammation as the cause of malignancy. British Journal of Cancer 2001, 85(4):473-483.

20. Gaida MM, Steffen TGe, Günther F, Tschaharganeh DF, Felix K, Bergmann F, Schirmacher P, Hänsch GM: Polymorphonuclear neutrophils promote dyshesion of tumor cells and elastase-mediated degradation of E-cadherin in pancreatic tumors. European Journal of Immunology 2012, 42(12):33693380 .

21. Bausch D, Pausch T, Krauss T, Hopt UT, Fernandezdelcastillo C, Warshaw AL, Thayer SP, Keck T: Neutrophil granulocyte derived MMP-9 is a VEGF independent functional component of the angiogenic switch in pancreatic ductal adenocarcinoma. Angiogenesis 2011, 14(3):235. 
22. Coffelt SB, Kersten K, Doornebal CW, Weiden J, Vrijland K, Hau C-S, Verstegen NJM, Ciampricotti M, Hawinkels LJAC, Jonkers $\mathrm{J}$ et al: IL-17-producing $ү \delta \mathrm{T}$ cells and neutrophils conspire to promote breast cancer metastasis. Nature 2015, 522:345.

23. Salazar-Onfray F, López MN, Mendoza-Naranjo A: Paradoxical effects of cytokines in tumor immune surveillance and tumor immune escape. Cytokine \& Growth Factor Reviews 2007, 18(1):171-182.

24. Qi C, Li B, Guo S, Wei B, Shao C, Li J, Yang Y, Zhang Q, Li J, He X: P-Selectin-Mediated Adhesion between Platelets and Tumor Cells Promotes Intestinal Tumorigenesis in ApcMin/+ Mice. International Journal of Biological Sciences 2015, 11(6):679-687.

25. Zhou Y, Cheng S, Fathy AH, Qian H, Zhao Y: Prognostic value of platelet-to-lymphocyte ratio in pancreatic cancer: a comprehensive meta-analysis of 17 cohort studies. Oncotargets \& Therapy 2018, 11:1899-1908.

26. Hasegawa S, Eguchi H, Tomokuni A, Tomimaru Y, Asaoka T, Wada H, Hama N, Kawamoto K, Kobayashi S, Marubashi S: Pre-treatment neutrophil to lymphocyte ratio as a predictive marker for pathological response to preoperative chemoradiotherapy in pancreatic cancer. Oncology Letters 2016, 11(2):1560-1566.

27. Martin HL, Ohara K, Kiberu A, Van Hagen T, Davidson A, Khattak MA: Prognostic value of systemic inflammation-based markers in advanced pancreatic cancer. Intern Med J 2014, 44(7):676-682.

28. Stotz M, Gerger A, Eisner F, Szkandera J, Loibner H, Ress AL, Kornprat P, Zoughbi WA, Seggewies FS, Lackner C: Increased neutrophil-lymphocyte ratio is a poor prognostic factor in patients with primary operable and inoperable pancreatic cancer. 2013, 109(2):416-421.

29. Yang JJ, Hu ZG, Shi WX, Deng T, He SQ, Yuan SG: Prognostic significance of neutrophil to lymphocyte ratio in pancreatic cancer: A meta-analysis. World J Gastroenterol 2015, 21(9):2807-2815.

30. Kishi T, Nakamura A, Itasaka S, Shibuya K, Matsumoto S, Kanai M, Kodama Y, Takaori K, Mizowaki T, Hiraoka M: Pretreatment C-reactive protein level predicts outcome and patterns of failure after chemoradiotherapy for locally advanced pancreatic cancer. Pancreatology 2015, 15(6):694-700.

31. Crown J, Casper ES, Botet J, Murray P, Kelsen DP: Lack of efficacy of high-dose leucovorin and fluorouracil in patients with advanced pancreatic adenocarcinoma. J Clin Oncol 1991, 9(9):1682-1686.

32. Van Rijswijk RE, Jeziorski K, Wagener DJ, Van Laethem JL, Reuse S, Baron B, Wils J: Weekly highdose 5-fluorouracil and folinic acid in metastatic pancreatic carcinoma: a phase II study of the EORTC Gastrolntestinal Tract Cancer Cooperative Group. Eur J Cancer 2004, 40(14):2077-2081.

\section{Tables}


Table 1. Characteristics of the 95 patients in study. Values refer to absolute number (n.) of patients and the corresponding percentage except for interval data. BMI: body mass index, KPS: Karnofsky performance status, CA 19-9: carbohydrate antigen 19-9, CEA:

carcinoembryonic antigen, CA-125: carbohydrate antigen-125, NLR: neutrophil-to-lymphocyte ratio. 


\begin{tabular}{|c|c|c|c|c|}
\hline Characteristics & $\begin{array}{c}\text { Category } \\
(\mathrm{n}=95)\end{array}$ & $\begin{array}{c}\mathrm{NLR} \leq 4.0 \\
(\mathrm{n}=68)\end{array}$ & $\begin{array}{c}\mathrm{NLR}>4.0 \\
(\mathrm{n}=27)\end{array}$ & $p$ value \\
\hline \multicolumn{5}{|l|}{ Gender } \\
\hline Male & $56(58.9)$ & $39(57.4)$ & $17(63.0)$ & \\
\hline Female & $39(41.1)$ & $29(42.6)$ & $10(37.0)$ & 0.586 \\
\hline \multicolumn{5}{|l|}{ Age } \\
\hline$\leq 65$ years & $66(69.5)$ & $46(67.6)$ & $20(74.1)$ & \\
\hline >65 years & $29(30.5)$ & $22(32.4)$ & $7(25.9)$ & 0.540 \\
\hline \multicolumn{5}{|l|}{ BMI } \\
\hline $\begin{array}{l}18.5-24.0 \\
\leq 18.5\end{array}$ & $\begin{array}{l}66(69.5) \\
12(12.6)\end{array}$ & $\begin{array}{c}48(70.6) \\
9(13.2)\end{array}$ & $\begin{array}{c}18(66.7) \\
3(11.1)\end{array}$ & \\
\hline$>24.0$ & $17(17.9)$ & $11(16.2)$ & $6(22.2)$ & 0.776 \\
\hline \multicolumn{5}{|c|}{ (2) (-) } \\
\hline$\leq 80$ & $40(42.1)$ & $26(38.2)$ & 14(51.9) & \\
\hline$>80$ & $55(57.9)$ & $42(61.8)$ & $13(48.1)$ & 0.225 \\
\hline \multicolumn{5}{|l|}{ History of Smoking } \\
\hline Yes & $31(32.6)$ & $19(27.9)$ & $12(44.4)$ & \\
\hline No & $64(67.4)$ & $49(72.1)$ & $15(55.6)$ & 0.122 \\
\hline \multicolumn{5}{|c|}{ History of Alcohol Intake } \\
\hline $\begin{array}{l}\text { Yes } \\
\text { No }\end{array}$ & $16(16.8)$ & $8(11.8)$ & $8(29.6)$ & \\
\hline \multicolumn{5}{|c|}{ Family History of Cancer } \\
\hline Yes & $13(13.7)$ & $9(13.2)$ & $4(14.8)$ & \\
\hline No & $82(86.3)$ & $59(86.8)$ & $23(85.2)$ & 0.840 \\
\hline \multicolumn{5}{|c|}{ Diabetes at Diagnosis } \\
\hline Yes & $31(32.6)$ & $23(33.8)$ & $8(29.6)$ & \\
\hline No & $64(67.4)$ & $45(66.2)$ & $19(70.4)$ & 0.694 \\
\hline \multicolumn{5}{|c|}{ 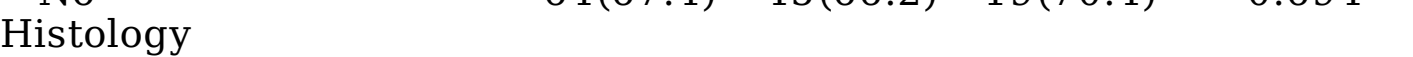 } \\
\hline PDA & 83(87.4) & $61(89.7)$ & $22(81.5)$ & \\
\hline Others & $12(12.6)$ & $7(10.3)$ & $5(18.5)$ & 0.276 \\
\hline \multicolumn{5}{|l|}{ T Stage } \\
\hline T1 & $8(8.4)$ & $7(10.3)$ & $1(3.7)$ & \\
\hline $\mathrm{T} 2$ & $31(32.6)$ & $20(29.4)$ & $11(40.7)$ & \\
\hline T3 & $28(29.5)$ & $20(29.4)$ & $8(29.6)$ & \\
\hline T4 & $28(29.5)$ & $21(30.9)$ & $7(25.9)$ & 0.590 \\
\hline \multicolumn{5}{|l|}{ N Stage } \\
\hline N0 & $25(26.3)$ & $23(33.8)$ & $2(7.4)$ & \\
\hline \multirow{2}{*}{\multicolumn{5}{|c|}{ M Stage }} \\
\hline MO & $26(27.4)$ & & & \\
\hline M1 & $69(72.6)$ & $43(63.2)$ & $26(96.3)$ & 0.001 \\
\hline \multicolumn{5}{|l|}{ Metastatic Site(s) } \\
\hline 0 & $26(27.4)$ & $25(36.8)$ & $1(3.7)$ & \\
\hline 1 & $37(38.9)$ & $26(38.2)$ & $11(40.7)$ & \\
\hline More than 1 & $32(33.7)$ & $17(25.0)$ & $15(55.6)$ & 0.001 \\
\hline \multicolumn{5}{|l|}{ Tumor Location } \\
\hline Head & $40(42.1)$ & $29(42.6)$ & $11(40.7)$ & \\
\hline Others & $55(57.9)$ & $39(57.4)$ & $16(59.3)$ & 0.865 \\
\hline \multicolumn{5}{|l|}{ CA $19-9$} \\
\hline$\leq 1000 \mathrm{U} / \mathrm{ml}$ & $56(58.9)$ & $41(60.3)$ & $15(55.6)$ & \\
\hline $\begin{array}{l}>1000 \mathrm{U} / \mathrm{ml} \\
\mathrm{CA}-125\end{array}$ & $39(41.1)$ & $27(39.7)$ & $12(44.4)$ & 0.672 \\
\hline \multicolumn{5}{|l|}{ CA-125 } \\
\hline $\begin{array}{l}\leq 38 \mathrm{U} / \mathrm{ml} \\
>38 \mathrm{U} / \mathrm{ml}\end{array}$ & $\begin{array}{l}39(41.1) \\
56(58.9)\end{array}$ & $34(50.0)$ & $12(44.4)$ & 0625 \\
\hline \multicolumn{5}{|l|}{ CEA } \\
\hline$\leq 5 \mathrm{ng} / \mathrm{ml}$ & $48(50.5)$ & $36(52.9)$ & $12(44.4)$ & \\
\hline$>5 \mathrm{ng} / \mathrm{ml}$ & $47(49.5)$ & $32(47.1)$ & $15(55.6)$ & 0.455 \\
\hline \multicolumn{5}{|l|}{ Therapy } \\
\hline $\mathrm{Su}$ & $12(12.6)$ & $6(8.8)$ & 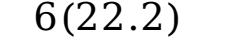 & \\
\hline
\end{tabular}


Table 2. Univariate and multivariate Cox-regression analysis for time to treatment failure and overall survival. Only significant results $(p<0.05)$ in the univariate analysis are run in the multivariate analysis. CI: confidence interval, HR: hazard ratio, TTF: time to treatment failure, OS: overall survival, BMI: body mass index, KPS: Karnofsky performance status, PDA: pancreatic ductal carcinoma, CA 19-9: carbohydrate antigen 19-9, CA-125: carbohydrate antigen-125, CEA: carcinoembryonic antigen, NLR: neutrophil-to-lymphocyte ratio, PLR: platelet-to-lymphocyte ratio, 5-FU:5-fluorouracil. 


\begin{tabular}{|c|c|c|c|c|c|}
\hline \multirow{2}{*}{$\begin{array}{l}\text { imber } \\
\text { th data }\end{array}$} & \multirow[t]{2}{*}{ n. $(\%)$} & \multicolumn{2}{|c|}{ Univariate analysis } & \multicolumn{2}{|c|}{ Multivariate analysis } \\
\hline & & TTF & OS & TTF & OS \\
\hline & & $\begin{array}{l}\text { HR }(95 \% \\
\text { CI), } p\end{array}$ & $\begin{array}{l}\mathrm{HR}(95 \% \\
\text { CI) }, p\end{array}$ & $\begin{array}{c}\mathrm{HR}(95 \% \\
\text { CI), } p\end{array}$ & $\begin{array}{l}\mathrm{HR}(95 \% \\
\mathrm{CI}), p \\
\end{array}$ \\
\hline & $\begin{array}{l}66(69.5) \\
29(30.5)\end{array}$ & $\begin{array}{l}1 \\
1.359(0.813- \\
2.272), \\
0.240\end{array}$ & $\begin{array}{l}1 \\
0.934(0.500- \\
1.742), \\
0.829\end{array}$ & & \\
\hline & $\begin{array}{l}56(58.9) \\
39(41.1)\end{array}$ & $\begin{array}{l}1 \\
0.754(0.465- \\
1.223), \\
0.251\end{array}$ & $\begin{array}{l}1 \\
0.666(0.357- \\
1.249), \\
0.203\end{array}$ & & \\
\hline$n^{2}$ & $66(69.5)$ & 1 & 1 & & \\
\hline 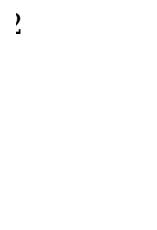 & $\begin{array}{l}17(17.9) \\
12(12.6)\end{array}$ & $\begin{array}{l}1.289(0.683- \\
2.433), \\
0.672(0.327- \\
1.382), \\
0.351\end{array}$ & $\begin{array}{l}1.958(0.944- \\
4.062) \\
0.694(0.268- \\
1.799), \\
0.100\end{array}$ & & \\
\hline & $\begin{array}{l}40(42.1) \\
55(57.9)\end{array}$ & $\begin{array}{l}1 \\
0.937(0.584- \\
1.502), \\
0.787\end{array}$ & $\begin{array}{l}1 \\
0.837(0.461- \\
1.520), \\
0.558\end{array}$ & & \\
\hline (5) & $\begin{array}{l}64(67.4) \\
31(32.6)\end{array}$ & $\begin{array}{l}1 \\
1.330(0.815- \\
2.171), \\
0.254\end{array}$ & $\begin{array}{l}1 \\
1.188(0.632- \\
2.231), \\
0.593\end{array}$ & & \\
\hline & $\begin{array}{l}79(83.2) \\
16(16.8)\end{array}$ & $\begin{array}{l}1 \\
1.462(0.811- \\
2.638), \\
0.207\end{array}$ & $\begin{array}{l}1 \\
1.564(0.767- \\
3.186), \\
0.215\end{array}$ & & \\
\hline & $\begin{array}{l}64(67.4) \\
31(32.6)\end{array}$ & $\begin{array}{l}1 \\
1.028(0.537- \\
1.968), \\
0.934\end{array}$ & $\begin{array}{l}1 \\
0.694(0.273- \\
1.762), \\
0.440\end{array}$ & & \\
\hline
\end{tabular}

35)

$\begin{array}{lll}40(42.1) & 1 & 1 \\ 55(57.9) & 1.068(0.649- & 1.022(0.549- \\ & 1.759), & 1.904), \\ & 0.796 & 0.945\end{array}$

5)

$$
\begin{array}{lll}
40(42.1) & 1 & 1 \\
55(57.9) & 0.860(0.533- & 0.834(0.458- \\
& 1.389), & 1.518), \\
& 0.538 & 0.551 \\
42(44.2) & 1 & 1 \\
53(55.8) & 1.430(0.891- & 1.215(0.670- \\
& 2.296), & 2.202),
\end{array}
$$$$
\text { ) }
$$ 
)
0.137
0.522

j)

$\begin{array}{lllll}25(26.3) & 1 & 1 & 1 & 1 \\ 70(73.7) & 2.271(1.276- & 3.225(1.419- & 1.422(0.757- & 3.180(1.288- \\ & 4.043), & 7.326), & 2.671), & 7.850), \\ & 0.005 & 0.005 & 0.273 & 0.012\end{array}$

)5)

$\begin{array}{lllll}26(27.4) & 1 & 1 & 1 & 1 \\ 69(72.6) & 3.715(1.981- & 5.359(2.067- & 2.148(0.997- & 1.306(0.410- \\ & 6.968), & 13.893), & 4.626), & 4.160), \\ & 0.0001 & 0.001 & 0.051 & 0.651\end{array}$

i)
83(87.4) 1
$12(12.6) \quad 1.417(0.741-1.452(0.643-$
2.710),
0.292
$3.281)$
0.369
56(58.9) 1
$39(41.1) \quad 1.868(1.142-\quad 2.987(1.608-$ 3.054),
0.013
$5.550)$
0.001
$\begin{array}{ll}1 & 1 \\ 1.434(0.815- & 1.981(0.953- \\ 2.526), & 4.117) \\ 0.212 & 0.067\end{array}$

46(48.4) 1

49(51.6) 1.999(1.230-

3.249),

1

0.005

2.209(1.176-

4.150),

0.014

48(50.5) 1

$47(49.5) \quad 1.714(1.062-$

2.765),

0.027

1

$3.112(1.639-$

5.906),

0.001

$\begin{array}{ll}1 & 1 \\ 1.393(0.832- & 1.382(0.710- \\ 2.333), & 2.688), \\ 0.207 & 0.341\end{array}$

$68(71.6) \quad 1$

$27(28.4) \quad 4.067(2.362-$

$7.002)$,

0.0001

1
$4.572(2.434-$
$8.589)$
0.0001

$1 \quad 1$

3.158(1.805- 4.090(2.073-

5.527),

8.071),

0.0001

0.0001

$\begin{array}{lll}56(58.9) & 1 & 1 \\ 39(41.1) & 1.511(0.940- & 1.683(0.917- \\ & 2.431), & 3.089), \\ & 0.088 & 0.089\end{array}$

)

-based 51(61.4) 1

$\begin{array}{lll}32(38.5) & 1.141(0.683- & 1.525(0.776- \\ & 1.908), & 2.995), \\ 0.614 & 0.217\end{array}$

Figures 
NLR

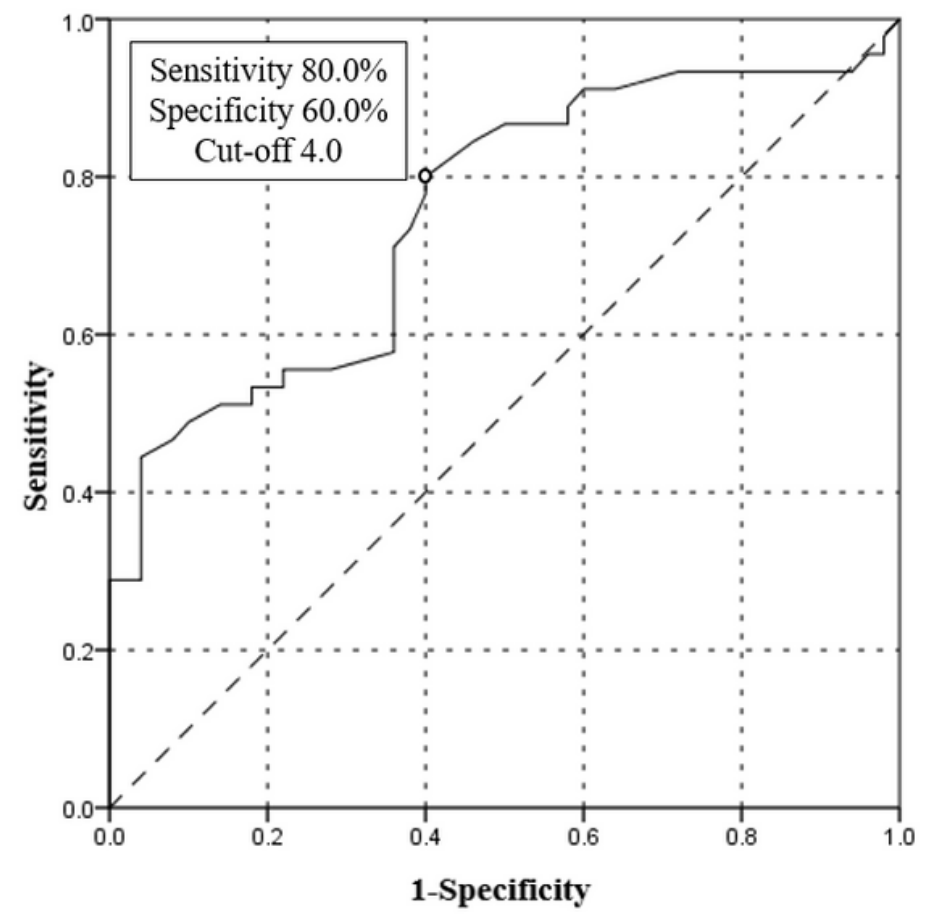

PLR

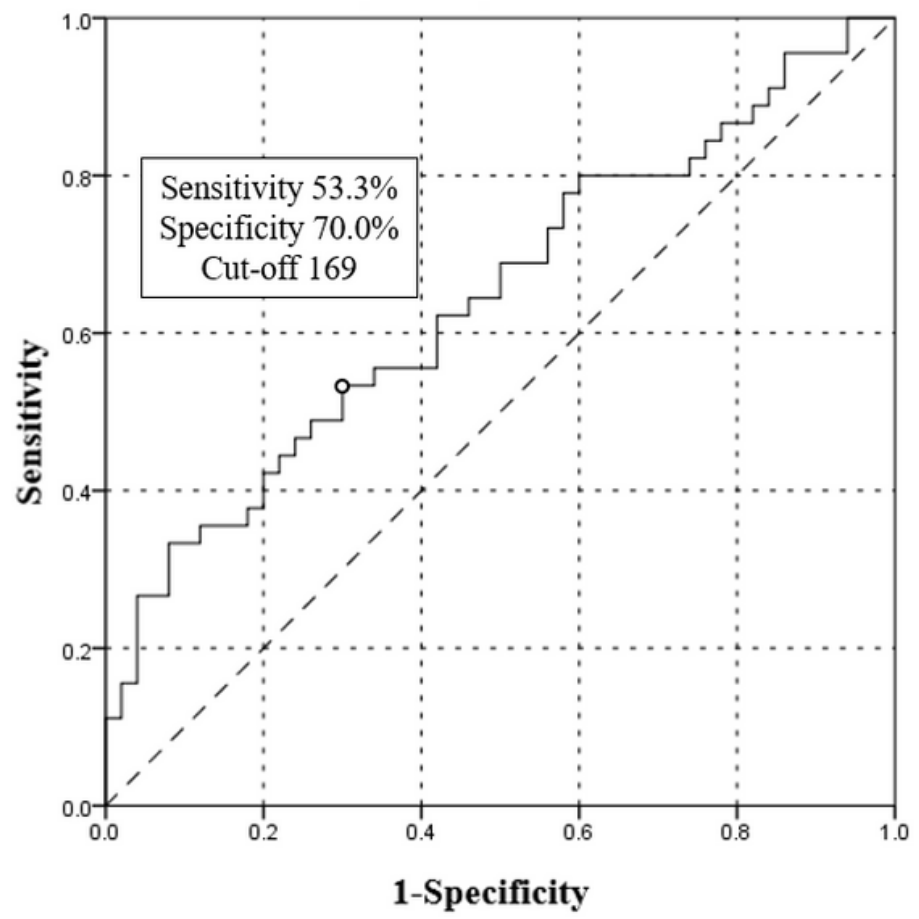

\section{Figure 1}

Receiver Operating Curve (ROC) analysis constructed to find the best cut-off point of neutrophil-tolymphocyte ratio (NLR) and platelet-to-lymphocyte ratio (PLR).
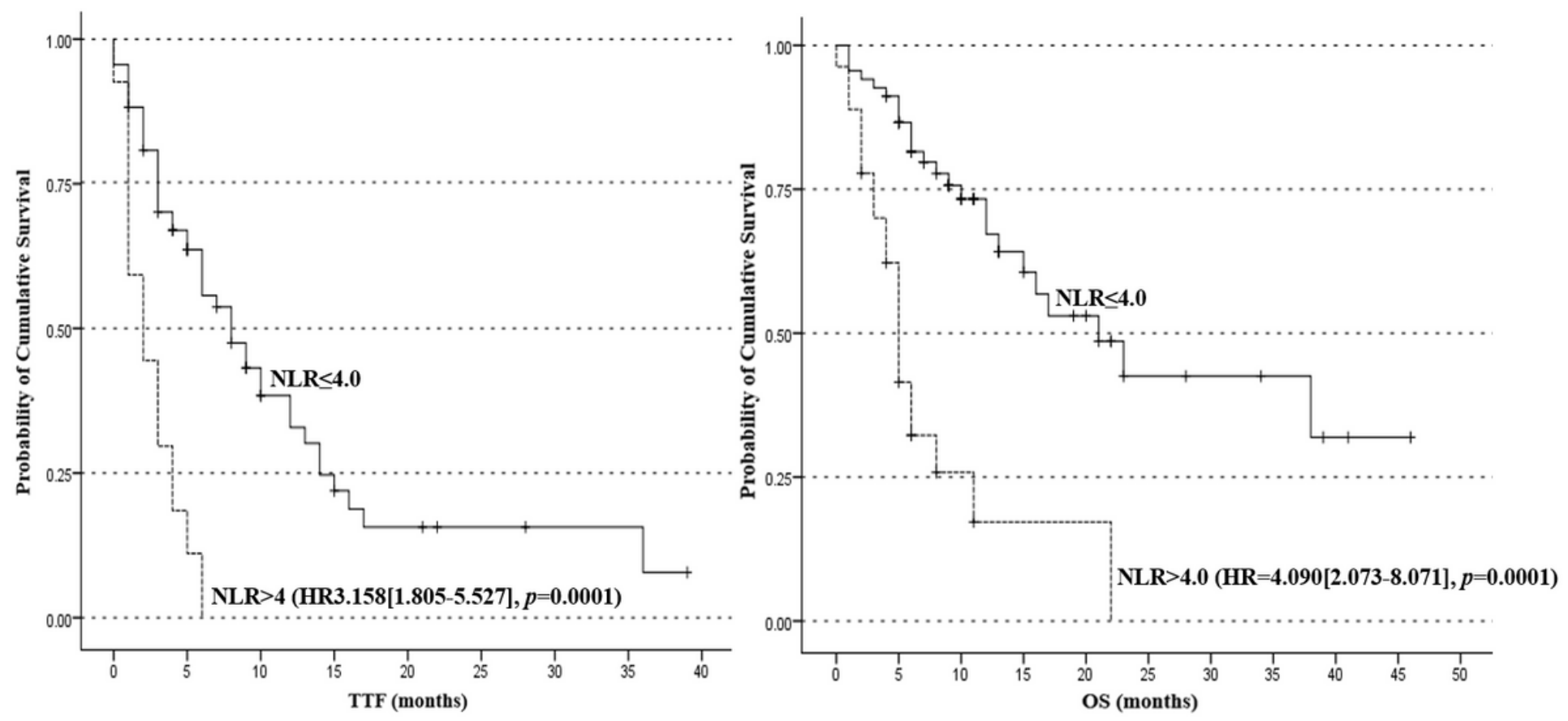

\section{Figure 2}

Time to treatment failure (TTF) and overall survival (OS) of patients classified by NLR $\leq 4.0$ or $>4.0$. 

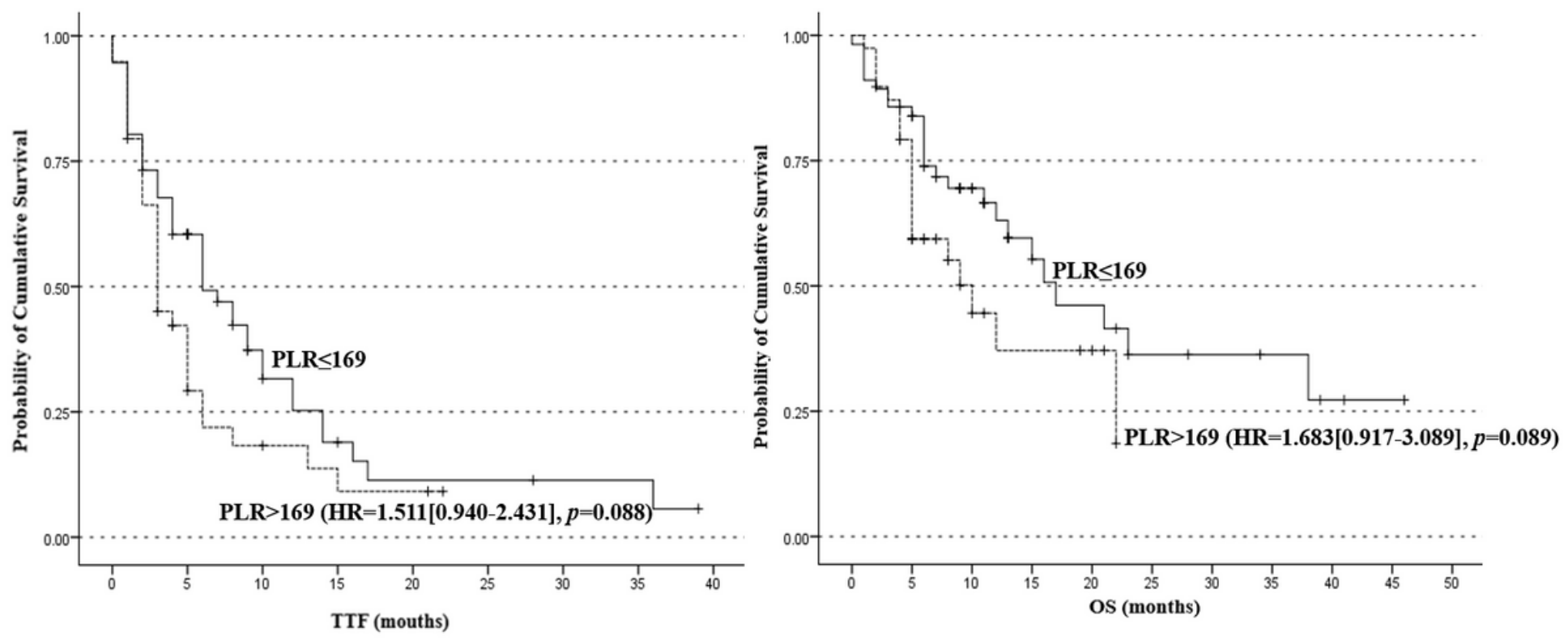

\section{Figure 3}

Time to treatment failure (TTF) and overall survival (OS) of patients classified by PLR $\leq 169$ or $>169$. 


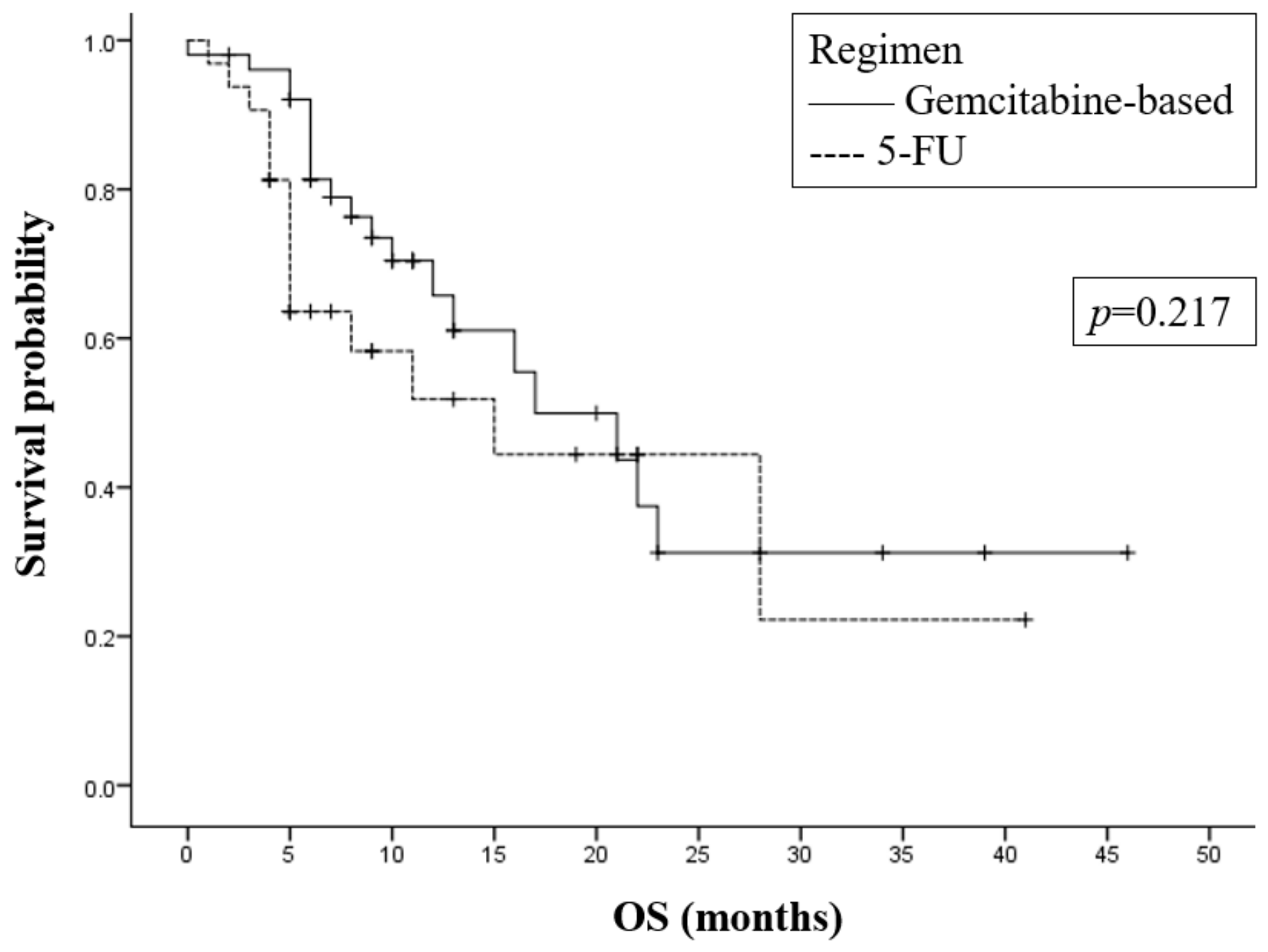

Figure 4

Overall survival (OS) and first-line chemotherapy regimen in 83 analyzed patients. 5-FU: 5-fluorouracil. 